\title{
OBJETO DE APRENDIZAGEM PARA APOIO AO PROCESSO ENSINO- APRENDIZAGEM DA TAXONOMIA NANDA
}

\author{
RIBEIRÃO PRETO/SP ABRIL/2018
}

\author{
Anicésia Cecília Gotardi Ludovino - IMEPAC - cecilialudo@hotmail.com \\ Leonardo Feriato Moreira - UNAERP - leoferiatom@yahoo.com.br \\ Silvia Sidnéia da Silva - UNAERP - sssilva@unaerp.br \\ Edilson Carlos Caritá - UNAERP - ecarita@unaerp.br
}

Tipo: Investigação Científica (IC)

Natureza: Relatório Final de Pesquisa

Categoria: Métodos e Tecnologias

Setor Educacional: EDUCAÇÃO SUPERIOR

\begin{abstract}
RESUMO
O objetivo do trabalho é apresentar o desenvolvimento de um Objeto de Aprendizagem (OA) para apoiar o processo ensino-aprendizagem de graduandos de enfermagem em relação a Assistência de Enfermagem por meio da Taxonomia North American Nursing Diagnosis Association (NANDA). Tratase de um estudo exploratório-descritivo com abordagem quantitativa. O OA é um app denominado iNANDA, desenvolvido por meio do framework Android Studio versão 3.0.1 com o Software Development Kit (SDK) do Android. O banco de dados foi elaborado utilizando o Real-Time Database versão 13.0 da plataforma de web service Firebase versão 13.0 da empresa Google. A validação do OA ocorreu com uma amostra constituída de 06 graduandos em enfermagem de uma Instituição de Ensino Superior privada mineira. A avaliação quantitativa visou analisar a eficácia do $O A$ no processo ensino-aprendizagem e a percepção do uso do OA pelos estudantes. Os dados foram analisados a partir do software Microsoft Excel 2016 e apresentados por meio de métricas de estatística. Os resultados demonstraram que $O O A$ contribuiu para o processo ensino-aprendizagem, pois antes de utilizar o app a média de acertos dos alunos na atividade avaliativa foi de $45,83 \% \pm 29,23 \%$ e após o uso do app a média de acertos atingiu $93,75 \% \pm 10,46 \%$. Também foi realizado o teste $t$, considerandose um $p$-value de $0,05(?=5 \%)$, o p-value do $P$ observado foi de $0,0046(0,46 \%)$, permitindo rejeitar a hipótese nula, pois o resultado indica que as médias das duas avaliações não são iguais estatisticamente, portanto, confirma a eficácia do OA. A maioria dos estudantes afirmou que o OA é de fácil uso e que não tiveram dificuldades para manuseá-lo, concordando unanimemente que o $O A$ contribuiu para seus conhecimentos, além de ser importante o uso da Tecnologia da Informação e Comunicação na educação. Conclui-se que o uso de OA para o ensino da Assistência em Enfermagem é uma ferramenta que auxilia e motiva o aluno em seu processo ensino-aprendizagem.
\end{abstract}

Palavras-chave: Objeto de Aprendizagem. Educação em Saúde. Tecnologia da Informação e Comunicação. Taxonomia NANDA. App.

AGRADECIMENTOS

AO CONSELHO NACIONAL DE DESENVOLVIMENTO CIENTÍFICO E TECNOLÓGICO (CNPQ) PELA BOLSA DE INICIAÇÃO CIENTÍFICA PIBITI. 


\section{INTRODUÇÃO}

O Processo de Enfermagem destaca-se como uma tecnologia do cuidado que orienta a sequência do raciocínio lógico e melhora a qualidade do cuidado por meio da sistematização da avaliação clínica, dos diagnósticos, das intervenções e dos resultados de enfermagem, constituindo numa ferramenta que deve ser utilizada pelos enfermeiros, pois evidencia o desencadeamento dos pensamentos e juízos desenvolvidos durante a realização dos cuidados, integra, organiza e garante a continuidade das informações da equipe de enfermagem, permitindo avaliar a sua eficácia e efetividade, modificando-a de acordo com os resultados na recuperação do cliente (DAL SASSO, 2013).

$\mathrm{Na}$ década de setenta, iniciou-se nos Estados Unidos da América (EUA) o movimento dos diagnósticos de enfermagem, tendo como finalidade, no princípio, a necessidade de explicitar para as seguradoras de saúde daquele país o que as enfermeiras realizavam na sua prática assistencial. Este movimento deu origem à North American Nursing Diagnosis Association (NANDA) a qual concebeu, então, a primeira taxonomia de diagnósticos de enfermagem, que se tornou a mais conhecida e utilizada mundialmente (BARROS; FAKIH; MICHEL, 2002).

A North American Nursing Diagnosis Association International - NANDA-I, que agrupa os Diagnósticos de Enfermagem (DE), pode ser utilizada em sistemas informatizados para a aplicação do Processo de Enfermagem, sendo um método que pode ser entendido como uma atividade intelectual deliberada, auxiliando a enfermeira na tomada de decisões, cujo foco reside na obtenção dos resultados esperados (SEGRANFREDO; ALMEIDA, 2011).

Nessa direção, atualmente, com o advento da Tecnologia da Informação e Comunicação (TIC), é comum a elaboração de OA utilizando recursos digitais para proporcionar a cooperação entre educação e saúde.

Os OA são recursos que estão sendo profusamente usados no processo ensinoaprendizagem dada suas contribuições como a versatilidade, dinamicidade e reusabilidade (REATEGUI; FINCO, 2010).

Sampaio e Almeida (2010) citam que os OA podem ser empregados desde a educação básica até cursos superiores, incluindo os da área da saúde, pois promovem a aprendizagem colaborativa, cuja ferramenta pedagógica permite a construção do conhecimento, contemplando a interação. 
Cabe ressaltar ainda que, segundo Tanaka et al. (2010), o uso da TIC como instrumento no processo ensino-aprendizagem vem ocorrendo de maneira expressiva nas instituições de ensino superior, portanto, os diversos cenários de educação, inclusive, no âmbito da educação em saúde, devem experimentar o uso de OA.

\section{OBJETIVO}

O objetivo desse estudo é apresentar o desenvolvimento de um Objeto de Aprendizagem (OA) para apoiar o processo ensino-aprendizagem de graduandos de enfermagem em relação a Assistência de Enfermagem por meio da Taxonomia North American Nursing Diagnosis Association (NANDA).

\section{MATERIAL E MÉTODOS}

Nessa seção apresentam-se os material e métodos utilizados no desenvolvimento do OA e a validação realizada por um grupo de estudante de graduação em enfermagem de uma Instituição de Ensino Superior (IES) privada do interior mineiro.

Para o desenvolvimento do OA foram realizadas pesquisas nas edições contemporâneas da Taxonomia NANDA e entrevistas com docentes enfermeiros de uma IES privada do interior paulista para o levantamento dos requisitos funcionais que foram contemplados no OA.

O OA é um app desenvolvido por meio do ambiente de desenvolvimento Android Studio versão 3.0.1 com o Software Development Kit (SDK) do Android e pode ser instalado em qualquer dispositivo que possua o sistema operacional Android na versão 4.4 (Android KitKat巴) ou superior.

Esse aplicativo recebe os dados contidos no banco de dados por meio de um web service, contudo, para que isso ocorra o app deve estar conectado à Internet.

O banco de dados foi elaborado utilizando o Real-Time Database versão 13.0 da plataforma de web serviceFirebase versão 13.0 da empresa Google.

O Real-Time Database é um serviço de web services oferecido na plataforma Firebase cuja principal característica é a atualização em tempo real dos dados, já cadastrados na base de dados, em todos os dispositivos que estiverem conectados a ele, por meio da Internet. Além disso, possibilita que esses dados fiquem armazenados nos dispositivos, permitindo que o app ainda seja totalmente funcional mesmo que não esteja conectado 
à Internet.

Segundo Lecheta (2015), web services são utilizados para fazer a integração entre sistemas, de modo que eles possam trocar dados. Isso permite que diferentes aplicações desenvolvidas em diferentes linguagens de programação possam se comunicar entre si por meio de um sistema distribuído, geralmente vinculado à rede mundial de computadores.

O web service envia os pedidos de requisição que a aplicação faz ao banco de dados que retorna ao web service os respectivos dados solicitados, o web service retorna os dados no formato JavaScript Object Notation (JSON), que é um dos formatos mais utilizados contemporaneamente para troca de informações entre sistemas de informação, e a aplicação consegue interpretar e aplicar esses dados aos seus processos.

O OA contempla informações sobre o diagnóstico de enfermagem da Taxonomia NANDA, permite realizar consultas aos diagnósticos de enfermagem cadastrados utilizando como palavras-chave o código, o título ou as características definidoras do diagnóstico.

Para validação da eficácia do OA no desempenho acadêmico, inicialmente, sem estudar com o OA, os estudantes resolveram um estudo de caso que versava sobre a definição do diagnóstico de enfermagem por meio da Taxonomia NANDA, e após quinze dias fazendo o uso OA, os alunos resolveram o mesmo estudo de caso para reavaliação de seus conhecimentos.

Os estudantes também responderam um instrumento estruturado com alternativas prédefinidas, com o objetivo de avaliar e apresentar subsídios para o aprimoramento do OA.

A amostra constituiu-se de seis graduandos de enfermagem de uma Instituição de Ensino Superior privada do Triângulo Mineiro, no município de Araguari, estado de Minas Gerais.

Para a análise quantitativa armazenou-se os dados coletados em uma planilha eletrônica do softwareMicrosoft Excel 2016 e os resultados foram apresentados por métricas estatísticas.

\section{RESULTADOS E DISCUSSÃO}


O app possui duas interfaces principais, a primeira é a tela inicial, onde são listados o código de identificação, o número da classe e do domínio a qual o diagnóstico pertence, bem como o título dos diagnósticos da Taxonomia NANDA, cadastrados na base de dados. O estudante de enfermagem que usar o app conseguirá acessar qualquer diagnóstico cadastrado. Para facilitar a consulta aos diagnósticos de enfermagem na tela há um botão com o ícone de uma lupa, e ao digitar o nome ou o código do diagnóstico é possível identificá-lo sem a necessidade de trocar de tela (Figura 1).

$\mathrm{Na}$ segunda tela é possível acessar uma explicação detalhada do diagnóstico de enfermagem selecionado na lista da tela inicial (Figura 2). Há um botão com um ícone de uma prancheta e ao clicá-lo ocorre o redirecionamento para outra tela, onde o estudante pode pesquisar o diagnóstico, informando no mínimo, três características definidoras (Figura 3). Na Figura 4 é apresentado o resultado de uma consulta realizada por meio das características definidoras.

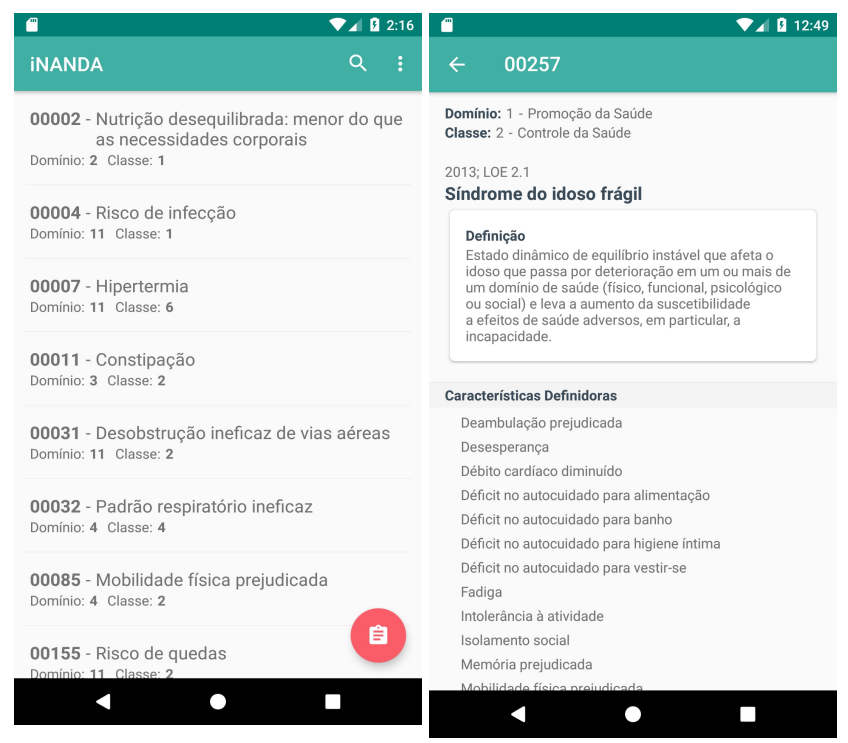

Figura 1 - Tela inicial

Figura 2 - Definição do diagnóstico de enfermagem Taxonomia NANDA 


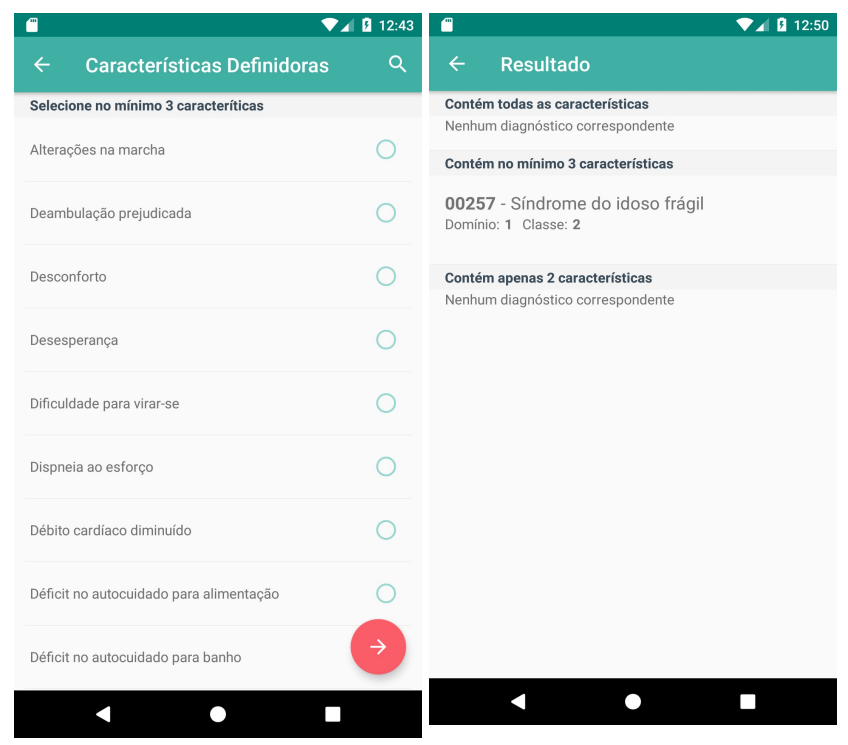

$\begin{array}{cc}\begin{array}{c}\text { Figura } 3-\text { Tela com as } \\ \text { características }\end{array} & \begin{array}{c}\text { Figura } 4-\text { Resultado } \\ \text { de Consulta pelas }\end{array} \\ \text { definidoras } & \text { características } \\ \text { definidoras }\end{array}$

Considerando os resultados da avaliação antes e após o uso do OA observa-se que, depois de utilizar o app, os alunos melhoraram consideravelmente seu desempenho acadêmico. O primeiro aluno antes do uso do OA teve nota zero e após o uso do OA obteve $75 \%$ de acertos; o segundo estudante inicialmente acertou $62,5 \%$ e depois de estudar com o OA acertou $100 \%$; o terceiro aluno atingiu $87,5 \%$ de acertos e manteve 0 mesmo indicador depois de fazer uso do OA; o quarto aluno, antes de usar o app acertou $50 \%$ e depois obteve $100 \%$ de acertos; já o quinto e o sexto alunos, a primeira vez fizeram corretamente $37,5 \%$ do estudo de caso e após o uso do OA acertaram $100 \%$.

No Gráfico 1 é apresentado um comparativo entre o desempenho dos estudantes antes e após uso do OA.

Gráfico 1 - Comparativo do antes e após uso do OA 


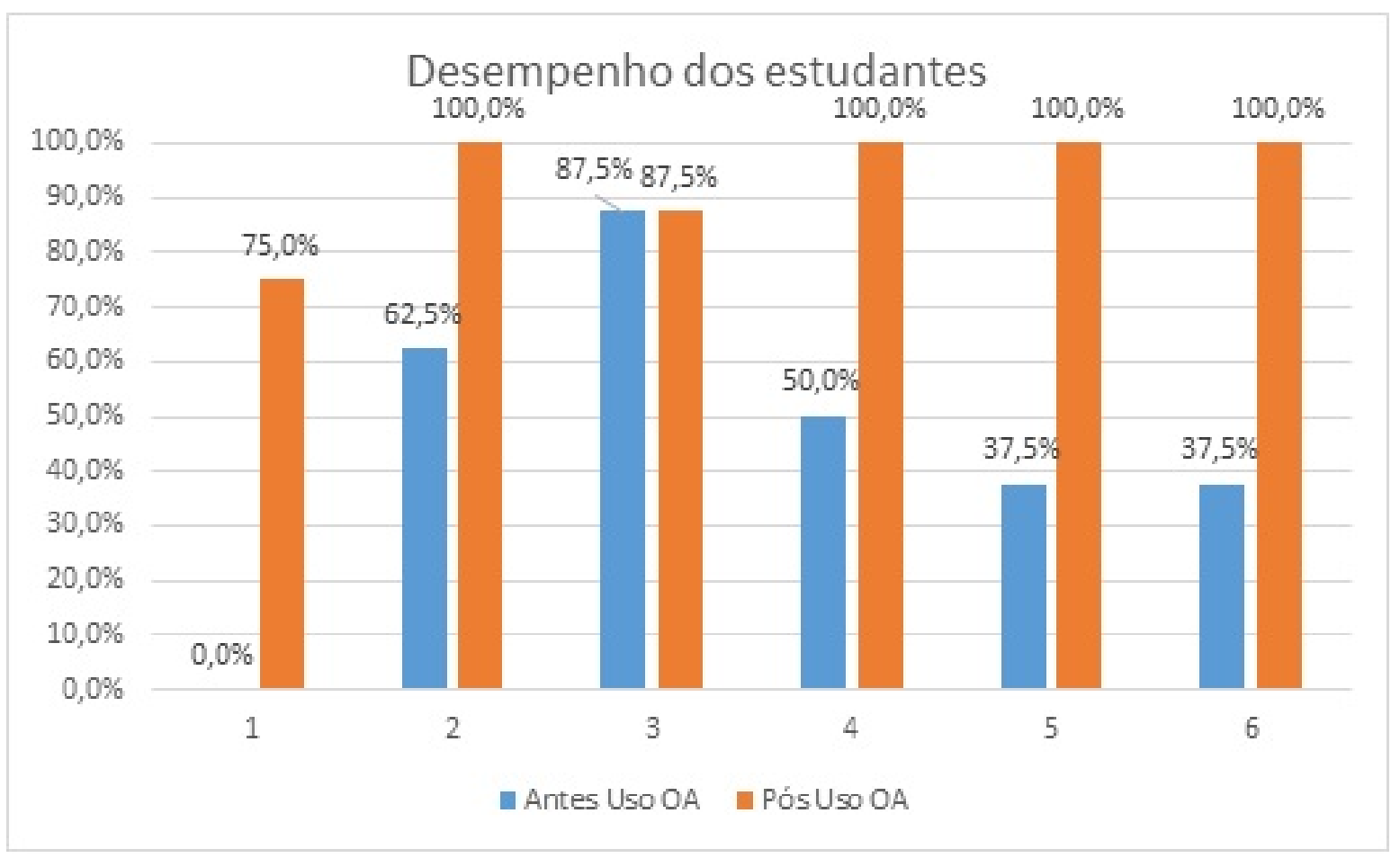

Fonte: Autoria Própria

Os resultados também demonstraram que antes de utilizar o app, a média de acertos dos alunos na resolução do estudo de caso foi de $45,83 \% \pm 29,23 \%$ e após o uso do app, a média de acertos atingiu 93,75\% $\pm 10,46 \%$. Também foi realizado o teste $t$, considerando-se um $p$-value de $0,05(?=5 \%)$, o $p$-value do $\mathrm{P}$ observado foi de 0,0046 $(0,46 \%)$, permitindo rejeitar a hipótese nula, portanto, o resultado indica que estatisticamente as médias das duas avaliações não são iguais e confirma a eficácia do OA.

A maioria dos alunos aprendeu a utilizar o OA no processo ensino-aprendizagem e obtiveram melhora no aprendizado da Taxonomia NANDA. Esse resultado corrobora com os achados de Alvarez e Dal Sasso (2011), pois salientam que os OA oferecem oportunidades no processo ensino-aprendizagem apresentando-se como recurso digital que pode ser utilizado para o suporte ao ensino propiciando ao graduando a construção do percurso cognitivo para aplicabilidade no processo ensino-aprendizagem em saúde e enfermagem.

Destaca-se ainda como resultado a avaliação da percepção dos estudantes quanto ao uso do OA desenvolvido para ser uma ferramenta de apoio ao processo ensinoaprendizagem. 
Na questão "Você teve alguma dificuldade em usar o OA?", 16,67\% responderam que tiveram dificuldade moderada, 50\% pouca e 33,33\% nenhuma. Assim, é possível afirmar que as interfaces do app são compreensíveis para $83,33 \%$ dos estudantes.

Em relação à pergunta "Depois de utilizar o OA você teve dificuldade de resolver o estudo de caso proposto?", 16,67\% disseram que tiveram muita dificuldade, 33,33\% pouca dificuldade e $50 \%$ nenhuma, demonstrando que o OA contribuiu no processo ensino-aprendizagem.

$\mathrm{Na}$ questão "O OA pode contribuir para sua aprendizagem em relação ao diagnóstico de enfermagem? "16,67\% concordaram e $83,33 \%$ concordaram totalmente. Desta maneira, infere-se que unanimemente os alunos concordaram que o OA desenvolvido é importante para o processo ensino-aprendizagem.

Considerando a questão "Como você avalia o conteúdo do OA?", 100\% responderam que o conteúdo é de fácil acesso e muito didático.

Quando perguntados "Você acha importante o uso da tecnologia da informação e comunicação na área da enfermagem?", 100\% assinalaram como concordo totalmente. Portanto, unanimemente, os participantes concordaram sobre a relevância da TIC no processo ensino-aprendizagem.

$\mathrm{Na}$ questão "Como você avalia a interação com o OA (acessar as informações)? "16,67\% manifestaram satisfatória, 33,33\% boa e 50\% muito boa. Observa-se, pelas respostas, que a maioria compreendeu a aplicabilidade do app como um recurso didático-pedagógico.

$\mathrm{Na}$ questão "Como você avalia a sua familiaridade com o OA?" 50\% manifestaram satisfatória, $16,77 \%$ boa e $33,33 \%$ muito boa; constatando-se pelas respostas, que a maioria demonstrou facilidade em utilizar a TIC no processo ensino-aprendizagem.

Corroboramos Cogo et al. (2009) ao relatarem que os objetos educacionais digitais promovem ousadia na busca de novos conhecimentos que capacitem os alunos de avançar na construção de sua própria aprendizagem tendo autonomia de pensamentos.

Vale ressaltar ainda que com a imersão da TIC na enfermagem é observada a necessidade de os profissionais buscarem atualização em conhecimentos adquiridos previamente com vistas ao desenvolvimento da educação e do cuidado de enfermagem (DAL SASSO; SOUZA, 2006). 


\section{CONCLUSÃO}

A maioria dos alunos concordou que a utilização do OA facilitou o processo ensinoaprendizagem relativo a Taxonomia NANDA, indicando, assim, que adotar OA como recurso didático-pedagógico nos cursos de graduação em Enfermagem nas modalidades presencial, educação a distância ou híbrido corrobora para a motivação dos alunos e apoia o desenvolvimento cognitivo para a competência referente à realização da assistência de enfermagem.

Conclui-se que o OA desenvolvido e disponibilizado na Google Play Store é uma ferramenta que tem aplicação no ensino do diagnóstico de enfermagem pela Taxonomia NANDA e pode contribuir para a formação de profissionais de enfermagem, em diferentes modalidades de ensino.

\section{REFERÊNCIAS BIBLIOGRÁFICAS}

ALVAREZ, Ana Graziela; DAL SASSO, Grace Teresinha Marcon. Objetos virtuais de aprendizagem: contribuições para o processo de aprendizagem em saúde e enfermagem. Acta Paulista de Enfermagem. Blumenau, v. 24, n. 5, p. 707-711, 2011.

BARROS, Alba Lucia Bottura Leite de; FAKIH, Flávio Trevisani; MICHEL, Jeanne Liliane Marlene. O uso do computador como ferramenta para a implementação do processo de enfermagem: a experiência do Hospital São Paulo/UNIFESP. Revista Brasileira de Enfermagem. Brasília, v. 55, n. 6, p.714-719, nov.dez.2002.

COGO, Ana Luísa Petersen et al. Objetos educacionais digitais em enfermagem: Avaliação por docentes de um curso de graduação. Revista da Escola de Enfermagem da USP. São Paulo, v.43, n. 2, p. 295-299, 2009.

DAL SASSO, Grace Teresinha Marcon et al. Processo de enfermagem informatizado: metodologia para associação da avaliação clínica, diagnósticos, intervenções e resultados. Revista da Escola de Enfermagem da USP. São Paulo, v. 47, n. 1, p. 242-249, 2013.

DAL SASSO, Grace Teresinha Marcon; SOUZA, Maria L. A simulação assistida por computador: a convergência no processo de educar-cuidar da enfermagem. Texto \& Contexto Enfermagem. Florianópolis, v. 15, n. 2, p. 231-239, 2006. 
Java na nuvem do Google. São Paulo: Novatec Editora Ltda., 2015.

REATEGUI, Eliseo; FINCO, Mateus David. Proposta de diretrizes para avaliação de objetos de aprendizagem considerando aspectos pedagógicos e técnicos. Revista Novas Tecnologias na Educação - RENOTE. Rio Grande do Sul, v. 8, n. 3, p. 1-10, 2010.

SAMPAIO, Romilson Lopes; ALMEIDA, Ana Rita Silva. Aprendendo matemática com objetos de aprendizagem. Ciências \& Cognição. Rio de Janeiro, v. 15, n. 1, p. 64-75, 2010.

SEGANFREDO, Deborah Hein; ALMEIDA, Miriam de Abreu. Validação de conteúdo de resultados de enfermagem, segundo a Classificação dos Resultados de Enfermagem (NOC) para pacientes clínicos, cirúrgicos e críticos. Revista Latino-Americana de Enfermagem. Porto Alegre, v. 19, n. 1, p. 19-28, 2011.

TANAKA, Raquel Yurika et al. Objeto educacional digital: avaliação da ferramenta para prática de ensino em enfermagem. Acta Paulista de Enfermagem. Porto Alegre, v. 23, n. 5, p. 603-607, 2010. 